\title{
Nonperturbative Renormalization for Domain Wall Fermions and the Chiral Condensate
}

\author{
Azusa Yamaguchi a

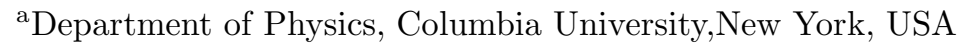

We study the chiral condensate, $\langle\bar{\psi} \psi\rangle$, and various quark bilinear vertex functions for domain wall fermions at different lattice scales, with both the Wilson and DBW2 gauge actions, in both quenched and dynamical fermion simulations. We use the vertex functions to calculate renormalization factors within a non-perturbative scheme.

\section{Introduction}

The RBC collaboration has performed simulations with domain wall fermions (DWF) and the DBW2 gauge action and presented results for weak matrix elements. This report focuses on the chiral condensate $\langle\bar{\psi} \psi\rangle$ and exploits nonperturbative renormalization calculations to get physical values for both quenched and dynamical fermions. The simulation details are given the Table 1 Two lattice volumes are used, $24^{3} \times 48$ for $a^{-1}=3 \mathrm{GeV}$, and $16^{3} \times 32$ for the others. Domain wall fermion are used for all simulations with three values of $L_{s}: 16$ for the Wilson gauge action and for the DBW2 gauge action with $a^{-1}=2 \mathrm{GeV}$ and $1.3 \mathrm{GeV}, 10$ for $a^{-1}=3 \mathrm{GeV}$ and 12 for dynamical fermions. Among these, data and simulation details for the Wilson gauge action and DBW2 gauge action with $1.3 \mathrm{GeV}$ and $2 \mathrm{GeV}$ have already reported 2]. The DBW2, $3 \mathrm{GeV}$ quenched and dynamical fermion simulations with $a^{-1}=$ $1.8 \mathrm{GeV}$ are reported in this meeting by $\mathrm{RBC}[3]$.

\section{2. $\langle\bar{\psi} \psi\rangle$ measurement}

Using DWF, we can compute the value of the chiral condensate directly by evaluating $\langle\bar{\psi} \psi\rangle$ at a series of quark mass and extrapolating to $m_{f}=-m_{\text {res }}$ (see the section 4). For the quenched, $a^{-1}=3 \mathrm{GeV}$ case, we use 5 fermion masses: $0.008,0.016,0.024,0.032,0.04$ and $m_{\text {res }}=9.72 \cdot 10^{-5}[3]$. For the dynamical case, three dynamical fermion masses $m_{f}^{d y n}=$ $0.02,0.03$ and 0.04 , and 5 valence masses, $m_{f}^{v a l}=$ $0.01,0.02,0.03,0.04$ and 0.05 are used. In the dy-
Table 1

Simulation details. Here "Q" means quenched while "D" means dynamical. The $3 \mathrm{GeV}$ simulation uses a $24^{3} \times 48$ volume while a $16^{3} \times 32$ volume is used for the others.

\begin{tabular}{lccl} 
Gauge action & Fermions & $L_{s}$ & $a^{-1}(\mathrm{GeV})$ \\
\hline Wilson & $\mathrm{Q}$ & 16 & $1.922(40)$ \\
DBW2 & $\mathrm{Q}$ & 16 & $1.97(4)$ \\
DBW2 & $\mathrm{Q}$ & 16 & $1.31(4)$ \\
DBW2 & $\mathrm{Q}$ & 10 & $2.86(9)$ \\
DBW2 & $\mathrm{D}$ & 12 & $1.80(7)$
\end{tabular}

namical case, in order to get a physical value, we extrapolate along the line $m_{f}^{v a l}=m_{f}^{d y n} \rightarrow$ $-m_{\text {res }}$. In the dynamical simulation, $m_{\text {res }}=$ $1.36 \cdot 10^{-3}$ 3. Besides the direct measurement of $\langle\bar{\psi} \psi\rangle$ above, we may also determine $\langle\bar{\psi} \psi\rangle$ from the $m_{f}$ dependence on the pion mass, using GellMann-Oakes-Renner (GMOR) relation:

$f_{\pi}^{2} \frac{m_{\pi}^{2}}{48\left(m_{f}+m_{\text {res }}\right)}=\langle\bar{\psi} \psi\rangle$

The values obtained using both methods are given in lattice units in Tab. 2. Apart from the $3 \mathrm{GeV}$ case, the values from each method agree nicely.

\section{Non-perturbative renormalization}

In order to get physical values for continuum observables, results obtained from lattice simulation need to be renormalized. The RBC group has used the non-perturbative RI renormalization scheme which benefits from the $\mathcal{O}(a)$ off-shell improvement of DWF. A detailed explanation of this method is given in the paper [4. There are two 
2

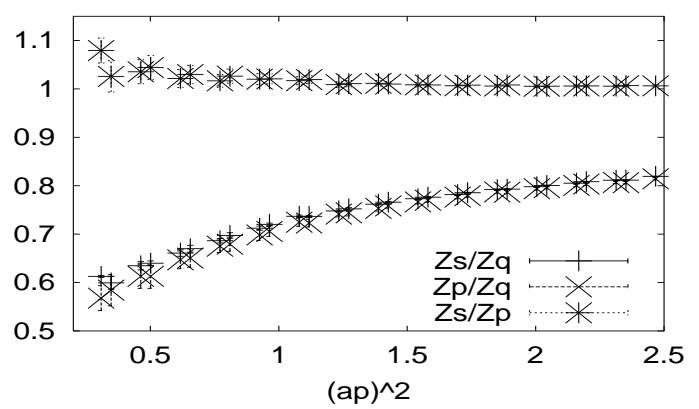

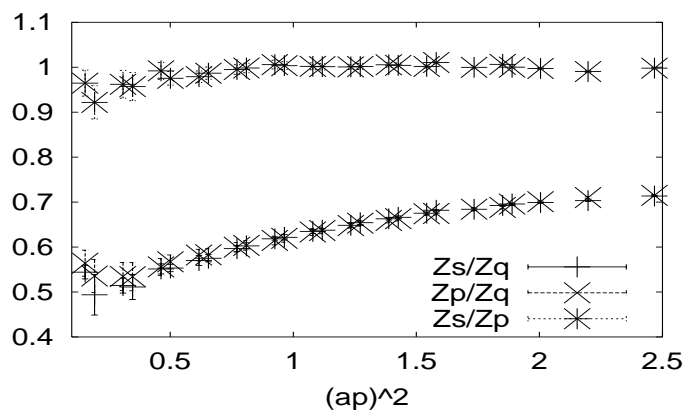

Figure 1. $Z_{S} / Z_{q}$ and $Z_{P} / Z_{q}$ plotted versus $p^{2}$ for $a^{-1}=3 \mathrm{GeV}$ (left) and dynamical fermions(right).

Table 2

Values of $\langle\bar{\psi} \psi\rangle$ from the GMOR relation, $\frac{b}{48} f_{\pi}^{2}$ and from direct measurement (the last column).

\begin{tabular}{lcllll} 
Action & $a^{-1}$ & $m_{f}$ & $b$ & $\frac{b}{48} f_{\pi}^{2}$ & $\langle\bar{\psi} \psi\rangle$ \\
\hline DBW2 quench DWF & $2 \mathrm{GeV}$ & $m_{f}>0.01$ & $2.58(2)$ & $2.3(2) \cdot 10^{-4}$ & $2.0(3) \cdot 10^{-4}$ \\
DBW2 quench DWF & $1.3 \mathrm{GeV}$ & $m_{f}>0.02$ & $5.02(41)$ & $1.00(2) \cdot 10^{-3}$ & $9.87(5) \cdot 10^{-4}$ \\
DBW2 quench DWF & $3 \mathrm{GeV}$ & $m_{f} \geq 0.008$ & $1.83(3)$ & $9.85(13) \cdot 10^{-5}$ & $1.78(2) 10^{-4}$ \\
DBW2 dynamical DWF & $1.75 \mathrm{GeV}$ & $m_{D} \geq 0.02$ & $3.70(10)$ & $5.12(91) \cdot 10^{-4}$ & $5.56(17) \cdot 10^{-4}$
\end{tabular}

systematic errors in this approach: lattice artifacts which appear at large momenta and nonperturbative phenomena which appear at small momenta. I will describe each of these in turn.

This approach gives renormalization factors for quark bilinear operators $\bar{u} \Gamma_{i} d,\left(\Gamma_{i}=\right.$ $\left.\left\{1, \gamma_{\nu}, \gamma_{5}, \gamma_{\nu} \gamma_{5}, \sigma_{\mu \nu}\right\}\right)$ where $u$ and $d$ are quark fields, using a corresponding vertex amplitude $\Lambda_{i}$. The renormalization factor $Z_{i}$ is determined by the condition:

$\left.Z_{\Gamma_{i}} Z_{q}^{-1} \Lambda_{\Gamma_{i}, 0}(p, p)\right|_{p^{2}=\mu^{2}}=1$.

For the case of $\Lambda_{S}$ and $\Lambda_{P}$, there are nonperturbative contributions which must be removed. They are shown in the equations:

$$
\begin{aligned}
\Lambda_{P, l a t t}(a p, a p) & =\frac{a^{2}\langle\bar{q} q\rangle}{(a p)^{2}\left(m_{f}+m_{r e s}\right)} C_{1} Z_{q} \\
& +Z_{m} Z_{q} \\
\Lambda_{S, l a t t}(a p, a p) & =\frac{C_{1} Z_{q}}{(a p)^{2}} \frac{\partial a^{3}\langle\bar{q} q\rangle}{\partial m_{f}}+Z_{m} Z_{q} .
\end{aligned}
$$

Subtracting the effects of the first term in each of these equations, gives values of $Z_{S} / Z_{P}$ close to unity over a large range of momentum, see Fig. 1]

Generally, renormalization factors in perturbation theory have logarithmic momentum dependence. This physical momentum dependence should be removed before attempting to identify
$\mathcal{O}\left(a^{2} p^{2}\right)$ errors. Therefore we define a scale invariant (SI) version of the RI renormalization factor:

$\Lambda_{\Gamma_{i}}^{S I}\left((a p)^{2}\right)=\Lambda_{\Gamma_{i}}\left((a p)^{2}\right) / C_{\Gamma_{i}}\left((a p)^{2}\right)$

where $C_{\Gamma_{i}}$ is calculated through three loops and is normalized so that $C_{\Gamma_{i}}\left(\mu^{2}\right)=1$. As an example, Fig. [2] shows the bare $Z_{S} / Z_{q}$ and its SI version for $3 \mathrm{GeV}$ and dynamical simulations. In this figure, the dashed line is a linear fit of $\Lambda_{\Gamma_{i}}^{S I}$ as a function of $(a p)^{2}$, permitting us to remove this large momentum lattice artifact.

To determine the conventional physical quantities defined in the continuum theory, it is necessary to convert renormalization factors obtained in RI-scheme to those in the $\overline{M S}$ scheme using a relation of the form:

$\frac{Z^{R I}}{Z^{\overline{M S}}}=1+\frac{\alpha_{s}}{4 \pi} Z_{0}^{(1) R I}+\frac{\alpha_{s}^{2}}{(4 \pi)^{2}} Z_{0}^{(2) R I}+\cdots$.

In order to extract the needed factor $Z_{S}$ and $Z_{P}$ from Eq. 2] we must determine $Z_{q}$. This is best done indirectly by using $Z_{A} / Z_{q}$ from the vertex function of the local axial current and $Z_{A}$ obtained from comparing the local and the conserved axial current. This method is described in detail in Ref. [1] and gives values for $Z_{A}$ of $0.8876(3)$ for $a^{-1}=3 \mathrm{GeV}$ and $0.7576(6)$ the dynamical case [3. Using these $Z_{A}$ value, $Z_{A} / Z_{q}$ and $Z_{S} / Z_{q}$ from RI-scheme and the Eq. B] we determine the $Z$ factors given in Tab. [4 

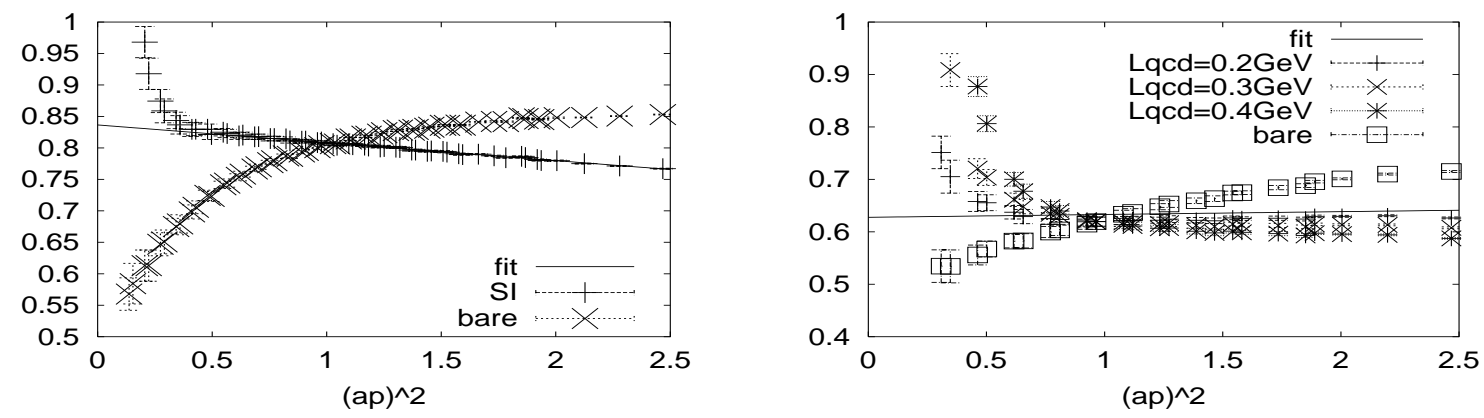

Figure 2. Bare and SI values $Z_{S} / Z_{q}$ plotted versus $p^{2}$ for $a^{-1}=3 \mathrm{GeV}$ (left) and dynamical fermions(right).

Table 3

Results for $\langle\bar{\psi} \psi\rangle$ expressed in physical units

$\begin{array}{cccccc}\text { Action } & a^{-1} & m_{\text {res }} & Z(\overline{M S}) & (12\langle\bar{\psi} \psi\rangle)^{1 / 3} & \left(12\left(\frac{b}{48} f_{\pi}^{2}\right)\right)^{1 / 3} \\ \text { DBW2 quench } & 1.31(4) & 5.7 \cdot 10^{-4} & 0.699(16) & 0.265(6) \mathrm{GeV} & 0.266(7) \mathrm{GeV} \\ \text { DBW2 quench } & 1.97(4) & 1.7 \cdot 10^{-5} & 0.712(14) & 0.235(13) \mathrm{GeV} & 0.246(9) \mathrm{GeV} \\ \text { DBW2 quench } & 2.86(9) & 9.72 \cdot 10^{-5} & 0.832(8) & 0.345(11) \mathrm{GeV} & 0.282(1) \mathrm{GeV} \\ \text { DBW2 dynamical } m_{D}=0.02 & 1.80(7) & 1.358 \cdot 10^{-3} & 0.469(6) & 0.263(10) \mathrm{GeV} & 0.253(18) \mathrm{GeV}\end{array}$

\section{4. $\langle\bar{\psi} \psi\rangle$ values in physical units}

Using the renormalization factors discussed in the previous section, we obtain the values for $\langle\bar{\psi} \psi\rangle$ in physical units given in Tab. [3 The results for $\langle\bar{\psi} \psi\rangle$ agree very well except for the direct calculation in the $3 \mathrm{GeV}$ case which is shifted $30 \%$ above the others. It is expected because of the $a$ dependence of the explicit chiral symmetry breaking in the domain wall scheme where $\langle\bar{\psi} \psi\rangle$ can be written in physical unit as,

$\langle\bar{\psi} \psi\rangle=c_{0} \Lambda_{Q C D}^{3}+\frac{c_{1}}{a^{2}} m_{f}+\frac{c_{2}}{a^{3}} e^{-L_{S} \alpha}$.

The last term in this equation can be estimated to be of the same size as $\frac{1}{a^{2}} m_{\text {res }}$. For the $\mathrm{GeV}$ case, this contributes an error to the value of $(12\langle\bar{\psi} \psi\rangle)^{1 / 3}$ quoted in Tab. 3 on the order of $20 \%$.

Table 4

Quenched $3 \mathrm{GeV}$ and dynamical $\mathrm{Z}$ factors. The dynamical results are given for $m_{f}^{d y n}=0.02$.

\begin{tabular}{lcc} 
Quantity & $3 \mathrm{GeV}$ quench & $1.75 \mathrm{GeV}$ dyn. \\
\hline RI\&SI $Z_{A} / Z_{q}$ & $0.962(9)$ & $0.916(6)$ \\
RI\&SI $Z_{S} / Z_{q}$ & $0.808(3)$ & $0.615(19)$ \\
$\overline{M S} Z_{A} / Z_{q}$ & $0.964(9)$ & $0.921(6)$ \\
$\overline{M S} Z_{S} / Z_{q}$ & $0.904(3)$ & $0.7366(98)$
\end{tabular}

This error is expected to decrease as $L_{s} \rightarrow \infty$.

\section{Conclusion}

Values for $\langle\bar{\psi} \psi\rangle$ are obtained from different methods with different lattice scales. Good agreement is found in all but one case where a larger $L_{s}$ is required.

\section{Acknowledgment}

I thank the RBC members for providing the simulation results analyzed in this report and many useful suggestions, especially Dr.C.Dawson for his help for NPR calculation, and Prof.N.Christ for his help.

\section{REFERENCES}

1. T. Blum, et al. $R B C$, hep-lat/0007038

2. Contributions of Y. Aoki, C. Dawson, J. Noaki and K. Orginos, to Lattice Conference '02. Nucl.Phys.B.(Proc.Suppl) Vol.119.

3. Contributions of Y. Aoki, C. Dawson, J. Noaki and K. Orginos, to Lattice Conference '03.

4. T. Blum, et al. $R B C$, hep-lat/0102005 